\title{
Enhanced tunnel conductance due to QCA cotunneling processes observed for silicon serial triple quantum dots
}

\author{
Gento Yamahata, Ken Uchida, and Shunri Oda \\ Quantum Nanoelectronics Research Center \\ Tokyo Institute of Technology, and SORST-JST \\ Tokyo, Japan \\ Email: ygent@neo.pe.titech.ac.jp
}

\author{
Yoshishige Tsuchiya and Hiroshi Mizuta \\ School of Electronics and Computer Science \\ University of Southampton \\ Southampton, U.K., \\ and SORST-JST, Tokyo Japan
}

\begin{abstract}
We study single-electron tunneling characteristics of silicon serial triple quantum dots which consist of lithographically-defined double quantum dots interconnected with a naturally-formed and smaller quantum dot. By controlling the single-electron tunneling through the triple quantum dots electrostatically using multiple side gates, the charge stability diagrams are characterized experimentally and theoretically. Several charge quadruple points are observed where sequential tunneling throughout the triple quantum dots is enabled . In addition, enhancement of tunnel conductance is observed along the two-hold degeneracy boundaries across which two electrons exhibit quantum cellular automata (QCA) cotunneling processes.
\end{abstract}

\section{INTRODUCTION}

In recent years, silicon double-quantum-dot (Si DQD) structures have been attracting much attention as a building block for quantum information devices (QIDs). Coherent manipulation of the qubit, the basic component of the QIDs, has been demonstrated mainly in compound semiconductor DQDs, for example, the electronic states qubit [1] and the electron spin qubit [2]. However, there exist several decoherence sources such as the hyper-fine interaction, the spin-orbit interaction, and the piezoelectric electron-phonon interaction. In silicon, these effects are presumably much smaller and the decoherence time of the qubits is expected to be improved. In addition, silicon qubits have a great advantage in integration because of the compatibility with the conventional silicon complementary metal-oxide-semiconductor (CMOS) processes. To realize the QIDs, the implementation of scalable multiqubit systems is necessary. For the first step toward the integration of the qubits, coherent manipulation of two qubits is required as well as that of one qubit. Hence, it is crucial to analyze the interaction between two qubits [3].

So far, we have investigated the electron transport through the individual Si DQDs and achieved the modulation of the electrostatic coupling in DQDs [4]. In this paper, we study the electron transport through the Si multiple quantum dot array devices (Si MQDADs) which have a pair of interconnected DQDs. We characterize the charge stability diagrams experimentally and theoretically, where fingerprints of a serial triple (a)

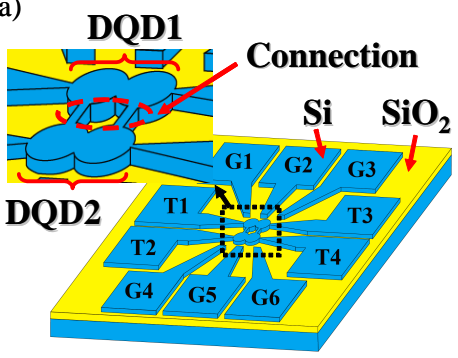

(b)

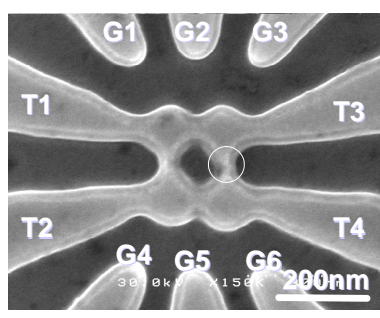

Fig. 1. (a) The schematic image of the silicon multiple quantum dot array devices (Si MQDADs). The two double quantum dots (DQD1 and DQD2) physically interconnected each other are connected to the four terminals (T1 T4). The side gates $(\mathrm{G} 1 \sim \mathrm{G} 6)$ located near the DQDs control the electrochemical potentials in the quantum dots (QDs). (b) Scanning electron microscope (SEM) image of the Si MQDAD.

quantum dots are identified. In addition, the enhancement of the tunnel conductance at the quantum cellular automata (QCA) lines clearly observed in experimental and simulated results.

\section{FABRICATION OF Si MQDADS AND INVESTIGATION OF FORMED QUANTUM DOTS}

Figure 1(a) shows the schematic image of the Si MQDADs. For enhancing electrostatic couplings between the two DQDs, DQD1 and DQD2 are physically interconnected each other. The MQDADs were defined in the silicon-on-insulator (SOI) of about $40 \mathrm{~nm}$ on the buried oxide (BOX) of $200 \mathrm{~nm}$ in thickness. First, the 40-nm-thick SOI film, whose thickness was reduced via thermal oxidation, was doped heavily by ion implantation (n-type, phosphorous, doping concentration $\sim 1 \times 10^{19} \mathrm{~cm}^{-3}$ ). The MQDADs were then patterned using high-resolution electron beam lithography (EBL) with the ZEP520A positive resist. The electron cyclotron resonance reactive ion etching was used to transfer the resist pattern onto the SOI layer, and $\mathrm{CF}_{4}$ was used as etching gas. Thermal oxidation was then done for $30 \mathrm{~min}$ at $1000{ }^{\circ} \mathrm{C}$ in order to passivate the surface states and reduce the dot size. Finally, 
(a)

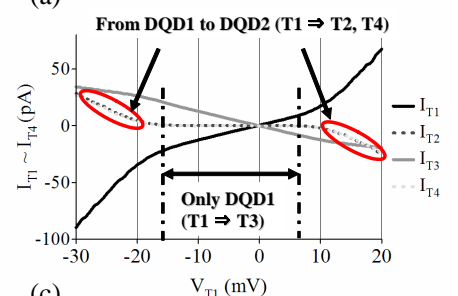

(c)

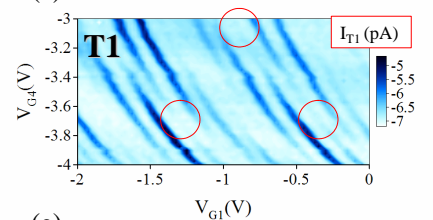

(e)

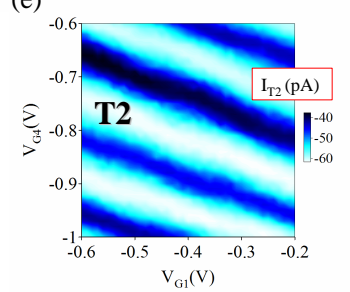

(b)
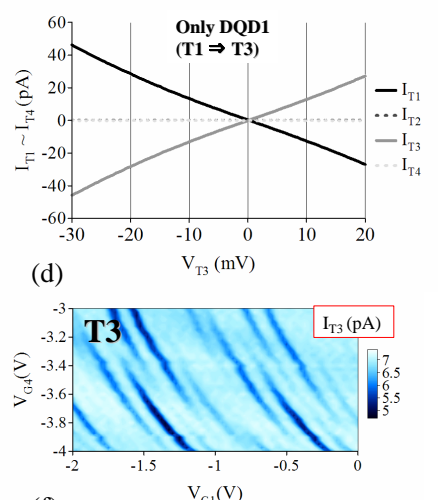

(f)

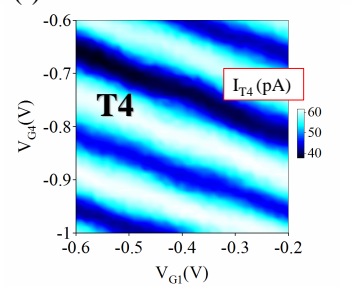

Fig. 2. The electrical characteristics for the investigation of the formed QDs in the MQDADs. (a, b) The currents $I_{T 1}, I_{T 2}, I_{T 3}$ and $I_{T 4}$, measured at the terminals T1, T2, T3, and T4, respectively, as a function of $V_{T 1}$ (a) and $V_{T 3}$ (b). (c, d) The contour plots of $I_{T 1}$ (c) and $I_{T 3}$ (d) as a function of $V_{G 1}$ and $V_{G 4}$, where $V_{T 1}$ is $-6 \mathrm{mV}$ and the other terminals are grounded. $(\mathrm{e}, \mathrm{f})$ The contour plots of the currents $I_{T 2}$ (e), and $I_{T 4}$ (f) as a function of $V_{G 1}$ and $V_{G 4}$, where $V_{T 1}, V_{T 2}$, and $V_{T 3}$ are $-6 \mathrm{mV}$ and $V_{T 4}$ is $0 \mathrm{mV}$.

Ohmic contacts were formed by evaporating about 300-nmthick Al. Figure 1(b) shows the scanning electron microscope (SEM) image of the Si MQDAD. In the lithographicallydefined strong constrictions, the bottom of the conduction bands rises due to the quantum-mechanical size effect, and, ideally, the eight constrictions should work as tunnel junctions.

We first studied the electrical connections among the quantum dots formed in the MQDADs. All measurements were performed by using the Hewlett Packard 4156A parameter analyzer at the temperature of $4.2 \mathrm{~K}$ in liquid helium. Figure 2(a) shows the currents $I_{T 1}, I_{T 2}, I_{T 3}$, and $I_{T 4}$, measured at the terminals T1, T2, T3, and T4, respectively, as a function of the voltage applied to $\mathrm{T} 1\left(V_{T 1}\right)$. Other terminals are grounded. In the low bias region $\left(-16 \mathrm{mV}<V_{T 1}<7 \mathrm{mV}\right)$, only $I_{T 1}$ and $I_{T 3}$ flow almost linearly. On the other hand, in the high bias region, $I_{T 2}$ and $I_{T 4}$ start to flow as indicated by the red circles in Fig. 2(a). In this region, the current path from T1 to T2 and T4 appears. The conduction path is presumably the left side interconnection between DQD1 and DQD2. However, when we apply the voltage only to T3 as shown in Fig. 2(b), only $I_{T 1}$ and $I_{T 3}$ flow. This is attributed to the weak, or almost disconnected right side interconnection, which is also supported by the SEM observation [shown by a circle in Fig. 1(b)].

Second, we characterized the current through DQD1 and DQD2. Figures 2(c) and 2(d) show the contour plots of $I_{T 1}$ and $I_{T 3}$ as a function of the gate voltages applied to the

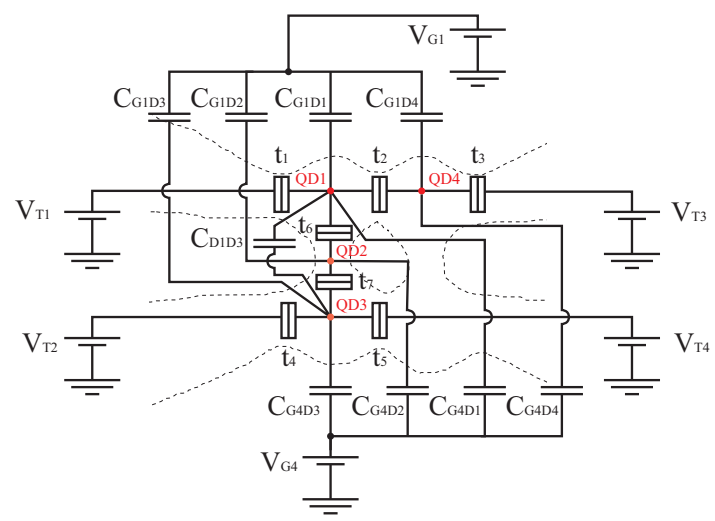

Fig. 3. The equivalent circuit of the MQDADs. The tunnel junctions $t_{a}$ $(a=1,2, \cdots, 7)$ consist of a tunnel resistance and a capacitor connected in parallel. $\mathrm{C}_{G b D c}(b=1,4, c=1,2,3,4)$ and $\mathrm{C}_{D 1 D 3}$ are the capacitors. $V_{T d}(d=1,2,3,4)$ are the bias voltage sources and $V_{G 1}$ and $V_{G 4}$ are the gate voltage sources. The dotted lines correspond to the SEM image of the MQDADs.

terminals $\mathrm{G} 1$ and $\mathrm{G} 4\left(V_{G 1}\right.$ and $\left.V_{G 4}\right)$, where $V_{T 1}$ is $-6 \mathrm{mV}$ and the other terminals are grounded. The bright regions of the contour plots correspond to the current peaks. In these gate voltage regions, the current flows only through DQD1. These current peak lines are not parallel and have the avoided crossings, for example, as indicated by the three red circles in Fig. 2(c). This result strongly suggest that DQDs with three tunnel junctions $\left(\mathrm{t}_{1}, \mathrm{t}_{2}\right.$, and $\mathrm{t}_{3}$ shown in Fig. 3$)$ are successfully realized within the lithographically-defined DQD1. On the other hand, the contour plots of $I_{T 2}$ and $I_{T 4}$ as a function of $V_{G 1}$ and $V_{G 4}$ exhibits current peak lines almost parallel with each other. This indicates that a single quantum dot (SQD) is responsible for the current oscillations. This is probably because the tunnel barrier between DQD2 and T4 is very low due to the weak constriction. Therefore only the left hand side quantum dot is active in DQD2 with the two tunnel barriers $\left(t_{4}\right.$ and $t_{5}$ shown in Fig. 3).

As a result of the above diagnosis, we found that our MQDAD device acts as a singly-interconnected DQD and SQD. However, for identifying actual quantum dots formed in the MQDADs, we have to take another possible mechanism of quantum confinement into consideration, which is the compressive stress generated during the pattern-dependent oxidation [5]. If a connection sandwiched with two large areas is very narrow, a small quantum dot is formed by the compressive stress accumulated in the connection. In the present device, the extremely narrow interconnection is likely to be the case as we see in the following analysis, and we assume an additional small quantum dot embedded in the interconnection region, resulting in serial triple quantum dots (see the equivalent circuit and superposed device geometry shown by dotted lines in Fig. 3).

\section{Electrical Characterization for the Si Serial TRIPLE QUANTUM DOTS IN THE MQDADS}

Figures 4(a)-4(d) show the contour plots of $I_{T 1}, I_{T 2}$, $I_{T 3}$, and $I_{T 4}$ as a function of $V_{G 1}$ and $V_{G 4}$, where $V_{T 1}$, 

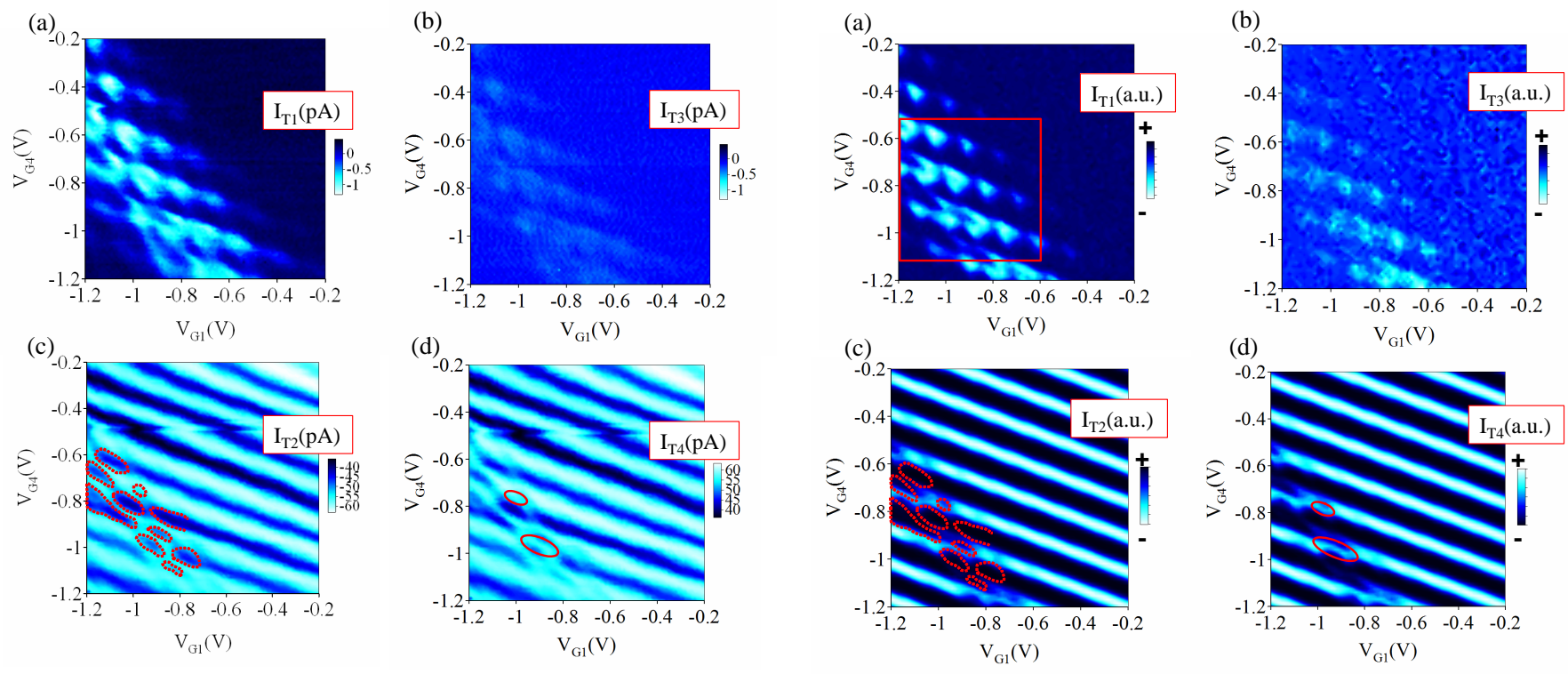

Fig. 4. The contour plots of $I_{T 1}$ (a), $I_{T 2}$ (b), $I_{T 3}$ (c), and $I_{T 4}$ (d) as a function of $V_{G 1}$ and $V_{G 4}$, where $V_{T 1}, V_{T 2}$, and $V_{T 3}$ are $-6 \mathrm{mV}$ and $V_{T 4}$ is $0 \mathrm{mV}$.

$V_{T 2}$, and $V_{T 3}$ are $-6 \mathrm{mV}$ and $V_{T 4}$ is $0 \mathrm{mV}$. We observed complicated current peaks with the various charge stability regions as indicated by the red doted lines in Fig. 4(c). We then simulated the electron transport through the MQDADs by using the equivalent circuit as shown in Fig. 3 with the Monte Carlo circuit simulator. Some circuit parameters are carefully extracted from the experimental results. The simulated stability diagrams as shown in Fig. 5 show an excellent agreement with the experimental results as shown in Fig. 4. The charge stability regions as indicated by the red dotted lines shown in Fig. 5(c) completely corresponds to those shown in Fig. 4(c). Since the electron transport through QD4 is almost suppressed in this bias region, the transport through the serial triple quantum dots (TQDs) which consist of QD1, QD2, and QD3, determines the entire transport characteristics. This is the first demonstration of the electron transport through the silicon serial TQDs. In the following, we will show the detail analysis of the transport through the TQDs.

In the case of serial DQDs [6], the electron stability diagrams are determined by the electrochemical potentials of the two quantum dots. There are two types of charging lines which have the different slopes, where the electrochemical potential for one particular electron configuration is zero. As the two charging lines cross, the intersection is avoided due to the electrostatic coupling and the pair of triple points appears, where the three charging configurations meet and the tunnel currents flow. As a result, the charge stability regions form the hexagonal shapes (the honeycomb diagram).

On the other hand, in serial TQDs [7], one more electrochemical potential for third quantum dot must be taken into account, leading to the third kinds of charging lines. If two of the three charging lines meet, the avoided crossing and the pair of triple points appear in a two-dimensional stability diagram

Fig. 5. The simulated contour plots of $I_{T 1}$ (a), $I_{T 2}$ (b), $I_{T 3}$ (c), and $I_{T 4}$ (d) as a function of $V_{G 1}$ and $V_{G 4}$, where $V_{T 1}=V_{T 2}=$ $V_{T 3}=-6 \mathrm{mV}$ and $V_{T 4}=0 \mathrm{mV}$. The circuit parameters are as follows: $t_{1}=t_{3}(C=16 \mathrm{aF}, R=500 \mathrm{k} \Omega), t_{2}(C=16 \mathrm{aF}, R=5 \mathrm{M} \Omega)$, $t_{4}=t_{5}(C=5.5 \mathrm{aF}, R=500 \mathrm{k} \Omega), t_{6}(C=1 \mathrm{aF}, R=1 \mathrm{M} \Omega)$, $t_{7}(C=0.5 \mathrm{aF}, R=1 \mathrm{M} \Omega), C_{G 1 D 1}=1.258 \mathrm{aF}, C_{G 1 D 2}=0.0178 \mathrm{aF}$, $C_{G 1 D 3}=0.409 \mathrm{aF}, C_{G 1 D 4}=0.05 \mathrm{aF}, C_{G 4 D 1}=0.546 \mathrm{aF}, C_{G 4 D 2}=$ $0.0136 \mathrm{aF}, C_{G 4 D 3}=1.097 \mathrm{aF}, C_{G 4 D 4}=0 \mathrm{aF}$ (because of very weak coupling), and $C_{D 1 D 2}=1 \mathrm{aF}$. Since $I_{T 3}$ is very small in the experiment results, we use high tunnel resistance for $t 2$. The Gate capacitances are estimated from experimental results. The offset gate voltages of $1.6 \mathrm{~V}$ for $V_{G 1}$ and $1.7 \mathrm{~V}$ for $V_{G 4}$ are used because of the background charges.

just as in the case of the DQDs. To obtain the full charging configurations in the TQDs, we need a three-dimensional stability diagram with three gates (the beehive diagram), where charging lines and triple points turn into charging plains and triple lines, respectively. In the crossing point of the two triple lines, which is referred to the quadruple point, the four electron configurations meet and the electron transports are allowed. However, the quadruple points are rarely observed because they are the distinct points in a three-dimensional space.

Our experimental and simulated results correspond to the two-dimensional slice of the beehive diagram. Since the measurements are performed at the nonlinear transport region, all the electrochemical potentials of QD1, QD2, and QD3 are allowed to enter the bias window not only at the quadruple points but also at the triple points in the vicinity of the quadruple points. Therefore the current peaks in Fig. 4(a) are attributed to be a few quadruple points and a lot of triple points. Far from quadruple points, the tunnel current peaks disappear [the right hand side of Fig. 4(a)] because of the electrochemical potentials in QD2 far from the bias window.

To discuss the electron configurations in the red square region in Fig. 5(a), we simulated the electron numbers $N_{1}$, $N_{2}$, and $N_{3}$ corresponding to those in QD1, QD2, and QD3, respectively, as shown in Fig. 6. Although our system has a lot of electrons because of the heavy doping concentration, we use the simple notation of the excess electron numbers as 


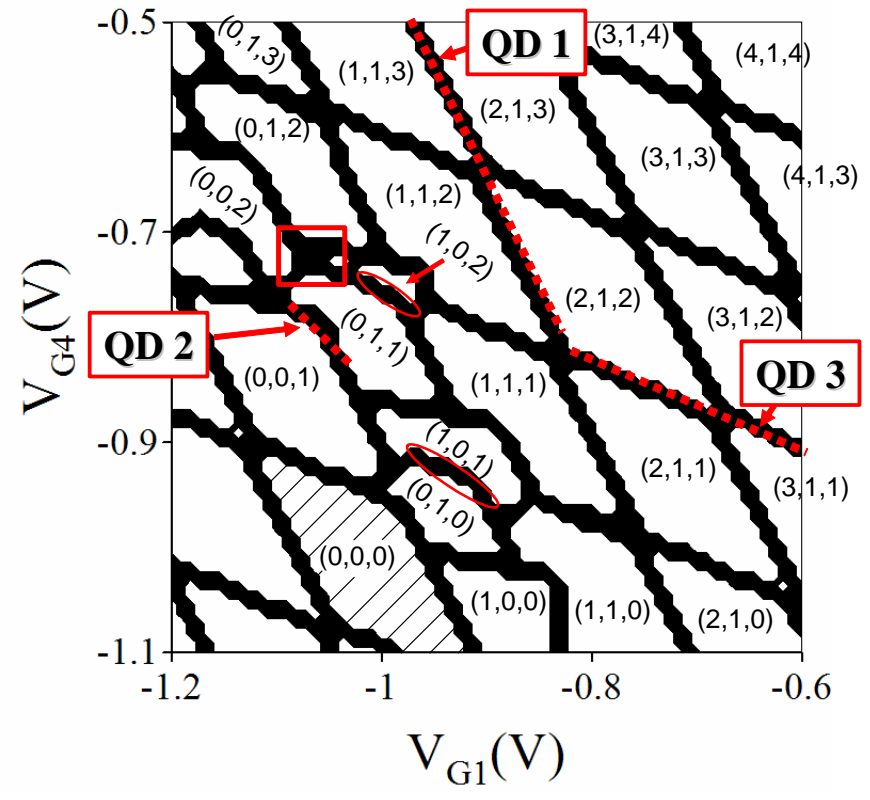

Fig. 6. The simulated electron numbers with the almost zero biases and at the temperature of $10 \mathrm{mK}$. The stable charge configuration in each domain is denoted by $\left(N_{1}, N_{2}, N_{3}\right)$, where $N_{1}, N_{2}$, and $N_{3}$ correspond to the electron numbers of QD1, QD2, and QD3, respectively. These electron numbers are not absolute values.

$\left(\mathrm{N}_{1}, \mathrm{~N}_{2}, \mathrm{~N}_{3}\right)=(0,0,0)$ at the hatched charge stability region. Here, the three types of charging lines with the different slops, corresponding to the electrochemical potentials of QD1, QD2 and QD3, are visible as indicated by the broken red lines in Fig. 6. At the other charging lines which have the other slopes, the transitions of the electrons at more than two QDs occur. In addition, the quadruple point appears in the simulated results (see the red square in Fig. 6). Here, the sequential tunneling occurs as $(0,1,1) \rightarrow(0,0,2) \rightarrow(1,0,2) \rightarrow(0,1,2)$, which involves two electrons in the transport.

Note that at the charging line between the stable electron configurations $\mathrm{A}=(0,1,0)$ and $\mathrm{B}=(1,0,1)$ as indicated by the lower red oval in Fig. 6, the electron numbers change at all QDs. Crossing the charging line from A to B, one electron moves from QD2 to QD3 (or 1) and simultaneously another electron enter QD1 (or 3). With the reverse process from B to $\mathrm{A}$, the two successive second order tunneling appears at this lines and the tunnel conductances are enhanced. These lines are referred to as quantum cellular automata (QCA) lines and two electrons exhibit QCA cotunneling processes [7]. The charging line as indicated by the upper red oval in Fig. 6 has also the same tunneling process. In the experimental and simulated results, the large currents at the QCA charging lines are observed as indicated by the red ovals in Fig. 4(d) and Fig. 5(d).

From the simulation circuit parameters, we can find the charging energies for QD1, QD2, and QD3 of $E_{Q D 1} \simeq$ $4.6 \mathrm{meV}, E_{Q D 2} \simeq 107 \mathrm{meV}$, and $E_{Q D 3} \simeq 8.7 \mathrm{meV}$, respectively. Note that since the charging energy of the QD2 is very large, the size of the QD2 is very small, whose diameter is estimated to be about $3.6 \mathrm{~nm}$ (assuming a cylindrical QD). From experimental results, we can estimates charging energies of the QD1 and QD3 of $E_{Q D 1} \simeq 7.7 \mathrm{meV}$ and $E_{Q D 3} \simeq$ $10.3 \mathrm{meV}$ from the nonlinear transport results. These values are almost consistent with those of the simulation results. The origin of the slight differences is probably due to the series resistances in the semiconductor leads. In addition, if we change the charging energy of the QD2 in the equivalent circuit, the simulated results dramatically change. Therefore, although it is difficult to accurately estimate the charging energy of the QD2 from our experimental results, the size of QD2 calculated by the simulation parameters is expected to be reasonable.

\section{CONCLUSION}

We fabricated the Si MQDADs with the high-resolution EBL technique and characterized their electron transport properties. The serial triple quantum dots forms by lithographically-defined double quantum dots interconnected with a naturally-formed and smaller quantum dot in the MQDADs. The experimental and simulated results show the electron transports through several triple points and quadruple points. Furthermore, the tunnel conductance is at the QCA charging lines are significantly enhanced with the second order tunneling processes. This is the first experimental observation of the QCA cotunneling processes for silicon nanostructures, which may be exploited for developing silicon-based quantum information processing devices.

\section{ACKNOWLEDGMENT}

This work was partly supported by KAKENHI (19.10625). One of authors, (G. Y.), is supported by the JSPS Research Fellowship for Young Scientists.

\section{REFERENCES}

[1] T. Hayashi, T. Fujisawa, H. D. Cheong, Y. H. Jeong, and Y. Hirayama, "Coherent Manipulation of Electronic States in a Double Quantum Dot," Phys. Rev. Lett. 91(22), 226804, 2003.

[2] F. H. L. Koppens, C. Buizert, K. J. Tielrooij, I. T. Vink, K. C. Nowack, T. Meunier, L. P. Kouwenhoven, and L. M. K. Vandersypen, "Driven coherent oscillations of a single electron spin in a quantum dot," Nature 442, 766, 2006.

[3] G. Shinkai, T. Hayashi, Y. Hirayama, and T. Fujisawa, "Controlled resonant tunneling in a coupled double-quantum-dot system," Appl. Phys. Lett. 90(10), 103116, 2007.

[4] G. Yamahata, Y. Tsuchiya, S. Oda, Z. A. K. Durrani, and H. Mizuta, "Control of Electrostatic Coupling observed for Silicon Double Quantum Dot Structures," to be published in Jpn. J. Appl. Phys.

[5] H. Horiguchi, M. Nagase, K. Shiraishi, H. Kageshima, Y. Takahashi, and K. Murase, "Mechanism of Potential Profile Formation in Silicon SingleElectron Transistors Fabricated Using Pattern-Dependent Oxidation," Jpn. J. Appl. Phys. 40(1A/B), pp. L29-L32, 2001.

[6] W. G. van der Wiel, S. De Franceschi, J. M. Elzerman, T. Fujisawa, S. Tarucha, and L. P. Kouwenhoven, "Electron transport through double quantum dots," Rev. Mod. Phys. 75(1), 1, 2003.

[7] D. Schröer, D. Greentree, L. Gaudreau, K. Eberl, L. C. L. Hollenberg, J. P. Kotthaus, and S. Ludwig, "Electrostatically defined serial triple quantum dot charged with few electrons," Phys. Rev. B 76(7), 075306, 2007. 